\title{
Concepts and Results in the Development of a Hybrid Tracking System for CAS
}

\author{
Wolfgang Birkfellner ${ }^{1 \star}$, Franz Watzinger ${ }^{2}$, Felix Wanschitz ${ }^{2}$, Georg Enislidis ${ }^{2}$, \\ Michael Truppe ${ }^{3}$, Rolf Ewers ${ }^{2}$ and Helmar Bergmann ${ }^{1,4}$ \\ 1 Department of Biomedical Engineering and Physics, University of Vienna \\ 2 Clinic of Oral and Maxillofacial Surgery, Medical School, University of Vienna \\ 3 ARTMA Medizintechnik, Vienna \\ 4 Ludwig-Boltzmann Institute of Nuclear Medicine, Vienna
}

\begin{abstract}
We present the design and the results achieved in the development of a hybrid magneto-optic tracking system suitable for computer aided surgery. Our approach towards a reliable and accurate hybrid position sensor system that is not entirely dependent on an unobstructed line-of-sight between sensor assembly and patient is the combined use of an optical tracker with a direct current (DC) pulsed electromagnetic tracking system (EMTS). The proposed hybridization method aims at providing both accurate and uninterrupted position data by overcoming the drawbacks of both tracking technologies. Results presented include the preliminary assessment of algorithms for fusion of position data from both sensor systems, for calibrating the EMTS to the environment and for detecting systematic distortions in the DC tracking system caused by ferromagnetic materials in order to improve the reliability of the proposed system.
\end{abstract}

\section{Introduction}

Navigation systems employed in computer aided surgery (CAS) are gaining increased acceptance as accurate and reliable tools for interventions where utmost accuracy is necessary [1]. While optical [2, 3, 4] and mechanical systems provide reliable position data, neither of these approaches can be considered perfect. Optical tracking systems (OTS) can be obstructed; the optical contact between patient and camera can break and cause a complete failure of the navigation device. This is not a severe drawback in interventions where the patient is immobilized by a Mayfield clamp or a similar device since only one instrument has to be tracked continuously. Most other interventions make it necessary to track both the patient and the tool. In interventions with a crowded operating field such as cranio- and maxillofacial surgery we consider the line-of-sight requirement to be a restriction forcing the surgeon to modify proven techniques. The usage of electromagnetic tracking systems (EMTS) which are not dependent on a free line-of-sight date back to the beginnings of CAS. The fact that the magnetic

\footnotetext{
* e-mail: wbirk@bmtp.akh-wien.ac.at
} 


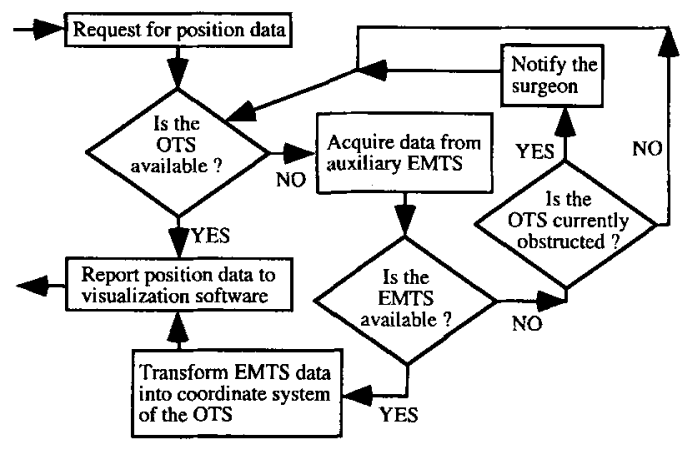

Fig. 1. A flow diagram of the hybrid tracking system. If the optical component of the system is unavailable due to obstruction of the optical path or low sampling rates of the OTS data from the auxiliary sensor system are employed and checked for validity. The methods for registration of the tracking systems and for assessing the data from the EMTS are presented in the paper.

field can be distorted by conductive or ferromagnetic materials in the vicinity of the sensor or the field emitter does, however, limit the usability of these devices for high precision surgical interventions. Experiments showed distortions caused by common surgical tools of $1.5-15 \mathrm{~mm}$ and $1-4^{\circ}$ [5]. Our approach towards a tracking system that overcomes these problems is to combine a direct current (DC) pulsed electromagnetic system with a highly accurate OTS. In order to ensure the reliability of such a hybrid tracker distortions in the reference field of the EMTS induced by ferromagnetic tools have to be noticed. Earlier work on sensor fusion for head tracking in augmented reality (AR) [6] lacks this feature.

In order to make a hybrid magneto-optic system usable for CAS the following criteria had to be fulfilled:

- assessment of method for registration of the tracking systems in order to

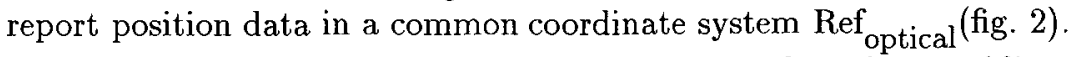

- development of calibration and registration algorithms for providing sufficient spatial accuracy of both tracking system components (error $\approx 1-1.5$ $\mathrm{mm}$ and $1^{\circ}$ in the operating theater).

- methods for ensuring the reliability of the electromagnetic tracker component by checking the validity of the position data acquired from the auxiliary EMTS.

A flow diagram of the data acquisition cycle can be seen in fig. 1. Once a request is being sent from the visualization software the system checks whether it can provide data from the OTS; if the OTS is not available, either due to obstruction of light emitting diodes (LED) or because of low sampling rates, the system requests position information from the electromagnetic component. In this case the hybrid system checks the validity of the readings and forwards the data, which have to be transformed to the coordinate system of the OTS only if the EMTS is not distorted. Therefore the system is able to handle periods of obstruction of the optical sensor component. Furthermore, the system's update rate is given by the sampling rate of the faster of the two systems when both trackers are in an operational state. Since the EMTS used is a DC pulsed system (the 


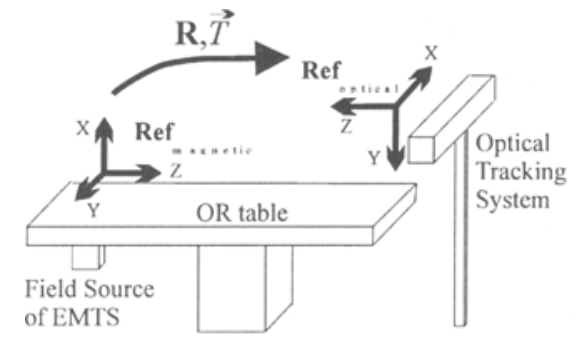

Fig. 2. Sketch illustrating the principle of the hybrid tracking system. The optical tracking system can be registered relative to the EMTS prior to a surgical intervention in order to obtain the transformation parameters $\mathbf{R}$ and $\mathbf{T}$, which merge the coordinate frames of the EMTS and the OTS.

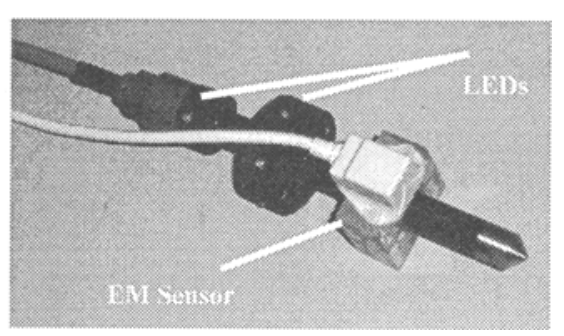

Fig. 3. Stylus setup used for the initial assessment of the hybrid tracking system. A stylus made of non- ferromagnetic materials (carbon fiber and titanium) was equipped with 16 LEDs and an electromagnetic sensor (EM Sensor).

magnetic reference field is produced by coils which are fed by rectangular currents rather than with alternating currents with continuously changing voltage) it is only susceptible to artifacts induced by ferromagnetic metals. Conductive materials do not affect the accuracy of the tracker [5] since the quasistatic magnetic field does not induce eddy currents in conductive materials once the field has been established.

\section{2 , Materials and Methods}

\subsection{Hardware}

The tracking systems used for our laboratory investigations are a widely used OTS (Flashpoint 5000, Image Guided Technologies Inc., Boulder, USA) with a spatial accuracy of $0.3-1.5 \mathrm{~mm}[2,3]$ and a DC driven EMTS (Flock of Birds, Ascension Technologies Inc, Colchester, USA). According to the specifications the nominal accuracy of the EMTS in an environment containing no ferromagnetic materials is given as $1.8 \mathrm{~mm} / 0.5^{\circ}$, and the spatial resolution is $0.5 \mathrm{~mm} / 0.2^{\circ}$. For data acquisition and implementation of the algorithms an IBM-PC running DOS 6.21 and Borland $\mathrm{C}++5.0$ was used. All software routines were implemented in ANSI-C.

\subsection{Clinical application and tool design}

The hybrid tracking system is planned for further refinement of techniques already in use at our hospital [7]. Fig. 3 shows the carbon fiber stylus which was used for our experiments. It contains 16 LEDs which ensure that position and orientation can be determined accurately by the OTS at all viewing angles. For laboratory tests we attached one EMTS sensor to the tool as shown (fig. 3) and 
calibrated the OTS to report the same position data in a common coordinate system as the magnetic sensor; this was achieved by acquiring position data from the EMTS sensor and determining an offset vector between a reference LED and the reported sensor position using the orientation data from the OTS and a Monte-Carlo optimization technique.

\subsection{Registration of the EMTS to the OTS}

Prior to calibration and intraoperative use the coordinate systems Ref magnetic and Ref ${ }_{\text {optical }}$ have to be registered relative to each other. The position readings from the OTS $\left(\mathbf{p}_{\text {opt }}\right)$ and the EMTS ( $\mathbf{p}_{\text {mag }}$ ) are merged by transforming $\mathbf{p}_{\text {mag }}$ to optical tracker space according to

$$
\hat{\mathbf{p}}_{\text {mag }}=\mathbf{R}_{\mathbf{p}_{\text {mag }}}+\mathbf{T}
$$

where $\hat{\mathbf{p}}_{\text {mag }}$ denotes the position data obtained from the EMTS in optical tracker space. The transformation parameters $\mathbf{R}$ (a rotation matrix) and $\mathbf{T}$ (the translation vector) were determined by applying a point-to-point registration algorithm based on singular value decomposition (SVD) [8]. The SVD-algorithm was taken from [9].

\subsection{Compensation of the influence from the OR environment on the EMTS}

For reasons to be discussed later the magnetic field emitter of the EMTS has to be placed below the OR table in a clinical setup. In a previous study on an acrylic skull model we found the distorting effect of the static OR-environment to be significant $\left(6.4 \pm 2.5 \mathrm{~mm} / 4.9 \pm 2.0^{\circ}\right)[5]$.

Therefore we implemented a calibration method for compensating the influence of the environment by using a distortion mapping technique $[10,11,12]$. A lookup table (LUT) was used to compensate the distortions caused by the static environment. This was achieved by acquiring accurate position information from the OTS and by correcting each measurement from the EMTS to the proper value. In our laboratory setup the digitizing volume of $200 * 200^{*} 200 \mathrm{~mm}^{3}$ was divided by a grid of $50 \mathrm{~mm}$ width. The stylus was placed to the grid positions in magnetic tracker space with an accuracy of $\pm 1.5 \mathrm{~mm}$. After placing the stylus at these positions the difference vector

$$
\boldsymbol{\Delta}_{i j k_{\mathrm{LUT}}}=\mathbf{R}^{\mathrm{T}}\left(\mathbf{p}_{\mathrm{opt}}-\mathbf{T}\right)-\mathbf{p}_{\mathrm{mag}}
$$

was determined. Before being reported to the visualization software the measurements from the EMTS have to be corrected and transformed to optical tracker space by calculating

$$
\mathbf{p}_{\text {mag }}^{\prime}=\mathbf{R}\left(\mathbf{p}_{\text {mag }}+\Delta_{i j k_{\text {LUT }}}\right)+\mathbf{T}
$$

The grid positions in magnetic tracker space are denoted by the indices $i, j$ and $k$. The grid point associated to a position $\mathbf{p}_{\text {mag }}$ is determined by seeking the closest 


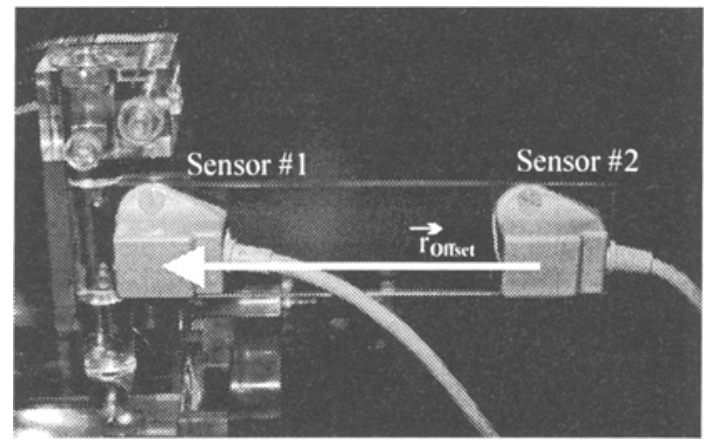

Fig. 4. Setup for detecting distortions in the EMTS. Two fluxgate sensors (Ascension Technologies Inc.) are mounted to a perspex plate (representing a rigid body) on a non-metallic frame. The difference between the true position of sensor \#1 and the measured value caused by a ferromagnetic sample was compared to the difference of the measured value $\left|\left\|\mathbf{x}_{1}-\mathbf{x}_{2}\right\|\right|$ from the known distance $\left\|\mathbf{r}_{\text {Offset }}\right\|$ of the sensors on the plate.

point on the calibration grid. The optimal value for $\boldsymbol{\Delta}_{i j k_{\text {LUT }}}$ was approximated using a linear interpolation.

A distortion mapping method meeting the high requirements of CAS also has to take into account the error in orientation data. However, calibrating the distortion of the orientation data for the EMTS is too difficult because of the number of measurements necessary. This is illustrated by the fact that authors in $[10,11,12]$ have not attempted to correct the orientation distortion. A possible solution to this problem is discussed.

\subsection{Automated Assessment of the Accuracy of Position Data obtained from the EMTS}

When using an EMTS in a surgical navigation device it is of vital importance to check the correctness of the position data acquired. Unlike the OTS, which fails in the case of obstruction but is highly accurate when a free line-of-sight to the camera bar is provided, the accuracy of the EMTS is affected by systematic errors caused by the high magnetic susceptibility of ferromagnetic materials and by a poor signal-to-noise ratio at greater distances from the emitter.

Our approach to ensure the reliability of acquired data without additional control from the OTS is to mount several sensors onto a rigid body (fig. 4). Due to the complex nature of the magnetic field the variation in field strength caused by a small body (i. e. a surgical tool) or by noisy readings is not shiftinvariant. Therefore comparing the known distance $\mathbf{r}_{\text {Offset }}$ on the rigid body to the measured apparent distance of two sensors in magnetic tracker space is a measure of the accuracy of the EMTS. 


\begin{tabular}{c|c|c|c|c}
\hline $\begin{array}{c}\text { EMTS } \\
\text { Readings }\end{array}$ & $\begin{array}{c}\overline{|\Delta x|} \\
{[\mathrm{mm}]}\end{array}$ & $\begin{array}{c}\overline{|\Delta y|} \\
{[\mathrm{mm}]}\end{array}$ & $\begin{array}{c}\overline{|\Delta z|} \\
{[\mathrm{mm}]}\end{array}$ & $\begin{array}{c}\overline{\Delta r} \\
{[\mathrm{~mm}]}\end{array}$ \\
\hline $\begin{array}{c}\text { Un- } \\
\text { corrected }\end{array}$ & $1.1 \pm 1.0$ & $2.0 \pm 1.0$ & $2.8 \pm 0.8$ & $3.9 \pm 0.9$ \\
\hline Corrected & $0.7 \pm 0.9$ & $1.1 \pm 0.8$ & $1.4 \pm 0.9$ & $2.2 \pm 1.0$ \\
\hline
\end{tabular}

Table 1. Average difference of measurements $(\mathrm{N}=125)$ from the distortion corrected and uncorrected EMTS data transformed to optical tracker space and the measurements from the OTS.

\section{Results}

\subsection{Calibration of the EMTS}

In a first experiment the readings from the EMTS were transformed to optical tracker space using the initial registration parameters $\mathbf{R}$ and $\mathbf{T}$ acquired from uncorrected readings $\mathbf{p}_{i_{\mathrm{mag}}}$. The corrected and uncorrected measurements $\left(\hat{\mathbf{p}}_{i_{\mathrm{mag}}}^{\prime}\right.$ and $\left.\hat{\mathbf{p}}_{i_{\mathrm{mag}}}\right)$ were compared to the data $\mathbf{p}_{i_{\mathrm{opt}}}$ acquired from the OTS, and both the translation errors $|\Delta x|,|\Delta y|$ and $|\Delta z|$ as well as the euclidean error $\Delta r$ were determined and averaged (table 1 ).

\subsection{Detection of Environmental Distortions}

The experimental setup shown in fig. 4 was used to determine the sensitivity of the proposed method for determining the reliability of the position readings from the EMTS with distorting materials like surgical tools present. The complete setup is described in more detail in [5]. The deviation induced by a steel rod (150 mm length, $10 \mathrm{~mm}$ diameter) from the known position of sensor \#1 was assessed by evaluating

$$
\Delta_{\text {det }}=||\left|\mathbf{x}_{2}-\mathbf{x}_{1}\left\|_{\text {measured }}-\right\| \mathbf{r}_{\text {Offset }} \|_{\text {known }}\right|
$$

where $\mathbf{x}_{1}$ and $\mathbf{x}_{2}$ denote the readings from sensor \#1 and \#2, and $\left\|\mathbf{r}_{\text {Offset }}\right\|$ is the known distance of the sensors on the rigid body as shown in fig. 4 . The difference between the fixed position of sensor \#1 and the acquired measurement $\mathbf{x}_{1}$ was compared to the quantity $\Delta_{\text {det }}$ (fig. 5). 100 measurements were taken and the difference between the true position of sensor \#1 and the acquired value $\mathbf{x}_{1}$ was plotted together with $\Delta_{\text {det }}$ as a function of the distance of the ferromagnetic sample to the sensor assembly. The values for the distance sample - sensor were collected into adjacent bins with a width of $50 \mathrm{~mm}$, and the ordinate values were averaged within these bins. It can be seen that an erratic reading of $\mathbf{x}_{1}$ also induces a deviation $\Delta_{\text {det }}$ which serves as a measure for the reliability of position data acquired.

In order to avoid artifacts due to Gaussian noise a threshold $\Delta_{\text {det }_{\text {thresh }}}$ for the deviation $\Delta_{\text {det }}$ was defined. If $\Delta_{\text {det }}$ exceeded this value the system was considered to be distorted. In $77 \%$ of all cases the EMTS was found to be distorted beyond a limit of $1.0 \mathrm{~mm}$. Several values for the threshold $\Delta_{\text {det }_{\text {thresh }}}$ were used $(0.5,1.0$, 1.5 and $2.0 \mathrm{~mm}$ ) for detecting these cases. The percentage of detected distortions 

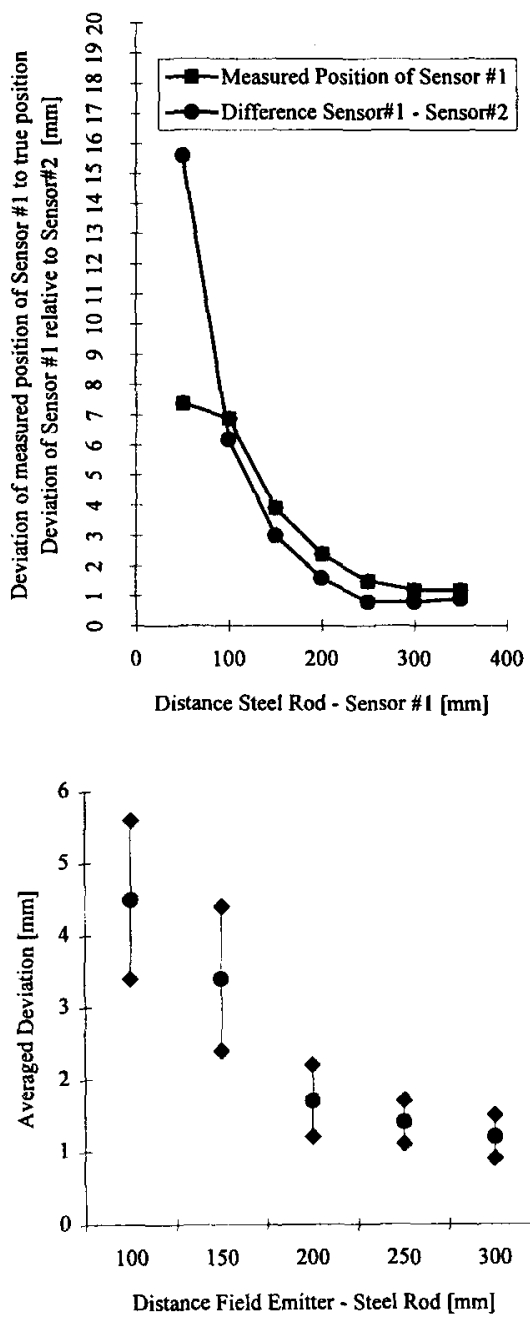

Fig. 5. Deviation from a constant position of electromagnetic sensor \#1 induced by a steel rod compared to the deviation of two sensors mounted to a perspex plate as shown in fig. 4. The displacement of the measured value from sensor \#1 and quantity $\Delta_{\text {det }}$ (eq. 4 ) are shown as a function of the distance between sensor assembly and ferromagnetic sample.
Fig. 6. Averaged error of EMTS sensor readings in cases where the distortion $\Delta_{\text {det }}$ caused by a steel sample exceede $1 \mathrm{~mm}$ and was not detected when using a threshold $\Delta_{\text {det }_{\text {thresh }}}$ of $1 \mathrm{~mm}$. Associated with each value is the percentage of the 25 cases where undetected distortion ocurred. The $\mathrm{x}$-axis denotes the distance of the ferromagnetic sample from the field emitter. The averaged true error is displayed together with its standard deviation.

as a function of $\Delta_{\text {det }_{\text {thresh }}}$ is presented in table 2 . From these results we concluded that a value of $1 \mathrm{~mm}$ would be optimal for $\Delta_{\text {det }_{\text {thresh }}}$ since the spatial resolution of the EMTS is given as $0.5 \mathrm{~mm}$.

In a further experiment we assessed the accuracy of the measured value $\left\|\mathbf{x}_{2}-\mathbf{x}_{1}\right\|_{\text {measured }}$ with and without LUT correction by moving the perspex plate shown in fig. 4 to 100 positions at random orientations (table 3 ). It can be seen that a value of $1.2 \mathrm{~mm}$ for $\Delta_{\text {det }_{\text {thres }}}$ was realizable in the laboratory experiments.

Furthermore we analyzed the cases where the error induced by the steel sample exceeded $1 \mathrm{~mm}$ and was not detected by the mechanism described above. It is straightforward to assume that the performance of the distortion detection methods is poor in cases where the distance from the sensor assembly and the ferromagnetic body exceeds the distance of the two electromagnetic sensors 


\begin{tabular}{c|c}
\hline$\Delta_{\text {det }_{\text {thresh }}}$ & $\begin{array}{c}\text { Detection rate } \\
\text { (in \%) }\end{array}$ \\
\hline 0.5 & 87 \\
1.0 & 67 \\
1.5 & 39 \\
2.0 & 28 \\
\hline
\end{tabular}

\begin{tabular}{c|c|c|c}
\hline $\begin{array}{c}\text { EMTS- } \\
\text { readings }\end{array}$ & $\begin{array}{c}\left\|\mathbf{r}_{1}-\mathbf{r}_{2}\right\| \\
{[\mathrm{mm}]}\end{array}$ & $\max$ & $\min$ \\
\hline $\begin{array}{c}\text { Uncorrected } \\
\text { Corrected }\end{array}$ & $102.7 \pm 1.0$ & 105.2 & 100.9 \\
\hline
\end{tabular}

Table 2. Capability of the experimental setup shown in fig. 4 to detect deviations from the true position of sensor \#1 beyond $1 \mathrm{~mm}$. Several thresholds $\Delta_{\text {det }_{\text {thresh }}}$ were allowed to assess the measured distance $\left|\left\|\mathbf{x}_{1}-\mathbf{x}_{2}\right\|\right|$ of the two sensors.

Table 3. Measured values in magnetic tracker space for the distance of two electromagnetic sensors mounted on a rigid body. The perspex plate was moved in the digitizer volume at random orientations and positions using calibrated and uncalibrated sensor readings.

significantly. Erratic readings from the EMTS are likely to occur if the ferromagnetic tool is exposed to high field strengths in the proximity of the emitter. The averaged difference between measured and true position of sensor \#1 was assessed as a function of the distance between steel sample and field emitter (fig. 6). A strategy for avoiding these situations is discussed.

\section{Discussion}

From our results we conclude that a tracking system with a guaranteed accuracy of less that $2 \mathrm{~mm}$ euclidean error is realizable by refining the methods for compensating static distortions and for detecting inaccuracies introduced by surgical instruments presented in this paper. Since several EMTS sensors have to be used, five or six degrees of freedom can be determined from the calibrated position readings only. In this configuration the problem of calibrating both position and orientation readings from the EMTS is avoided.

In order to improve the detection rate of invalid readings from the EMTS (fig. 6) it is necessary to physically block the emitter from the events in the operating theater. Mounting the emitter below the OR table and applying the distortion mapping routine appears to be a method for achieving this. Since the magnetic field used for position determination is very weak the operating range of the EMTS is delimited to a sphere of less than one meter in diameter. Outside this volume we have found the influence of the OR inventory to be negligible [5].

A user-friendly surgical tracking system should also be easy to be set up. Our method to perform this is to place the field emitter in a fixed position below the OR table. The influence of the ferromagnetic environment therefore has to be compensated once, whereas the optical component is registered relative to the calibrated EMTS before each intervention by using the methods described.

Some drawbacks of the current design include the difficulty of realizing wireless tracking like in videometric systems [4] although the rather huge mumber of signal leads from the single magnetometers can be reduced by multiplexing the 
raw sensor readings. Changing the geometry of the OR-table (for instance by tilting parts of it) would also destroy the registration of the setup.

We conclude that the development of hybrid tracking technologies has the potential to provide a reliable and user-friendly tracking system which promotes the integration of CAS technologies in demanding clinical routine applications.

\section{Acknowledgment}

We wish to thank A. Gamperl and W. Piller for their help in designing and manufacturing the hardware necessary for the experiments as well as Len Chamberlain (Image Guided Technologies Inc.) and Steve Work (Ascension Technologies Inc.) for their kind advice. Part of this work was supported by the Austrian Science Foundation FWF (Research Grant No. P12464-MED).

\section{References}

1. R. H. Taylor, S. Lavallée, G. C. Burdea, R. Mösges (eds.): "Computer-Integrated Surgery - Technology and Applications", MIT Press, Cambridge, (1996).

2. S. A. Tebo, D. A. Leopold, D. M. Long et al.: "An Optical 3D Digitizer for Frameless Stereotactic Surgery", IEEE Comput Graph, 55 - 64, (1996).

3. W. E. L. Grimson, G. J. Ettinger, S. J. White et al.: "An Automatic Registration Method for Frameless Stereotaxy, Image Guided Surgery, and Enhanced Reality Visualization", IEEE T Med Imaging, Vol. MI 15(2), 129-140, (1996).

4. A. C. F. Colchester, J. Zhao, K. S. Holton-Tainter et al.: "Development and preliminary evaluation of VISLAN, a surgical planning and guidance system using intraoperative video imaging", Med Image Anal, Vol. 1(1), 73-90, (1996).

5. W. Birkfellner, F. Watzinger, F. Wanschitz et al.: "Systematic Distortions in Magnetic Position Digitizers", paper accepted for publication in Med Phys.

6. A. State, G. Hirota, D. T. Chen et al.: "Superior Augmented Reality Registration by Integrating Landmark Tracking and Magnetic Tracking", Proceedings of SIGGRAPH 96, in Computer Graphics Proceedings, ACM SIGGRAPH, 429 - 438, (1996).

7. F. Watzinger, F. Wanschitz, A. Wagner et al.: "Computer-Aided Navigation in Secondary Reconstruction of Post- Traumatic Deformities of the Zygoma", J. Craniomaxillofac. Surg. 25, $198-202$, (1997).

8. K. S. Arun, T. S. Huang, S. D. Blostein, "Least- Squares Fitting of Two 3-D Point Sets", IEEE T Pattern Anal Vol. PAMI-9(5), 698-700, (1987).

9. W. H. Press, S. A. Teukolsky, W. T. Vetterling, B. P. Flannery: "Numerical Recipes in C - The Art of Scientific Computing, Second Edition", Cambridge University Press, (1992).

10. S. Bryson: "Measurement and calibration of static distortion of position data from 3D trackers", SPIE Vol. 1669, 244 - 255, (1992).

11. M. Ghazisaedy, D. Adamczyk, D. J. Sandin et al.: "Ultrasonic Calibration of a Magnetic Tracker in a Virtual Reality Space", in VRAIS '95, March 11-15, 1995, Proceedings, $179-188$, (1995).

12. M. A. Livingston, A. State: "Magnetic Tracker Calibration for Improved Augmented Reality Registration", Presence Vol. 6(5), 532 - 546, (1997). 